\title{
Parallel Video Steganographic Method over Multi-core Processors
}

\author{
Sally Almanasra \\ Faculty of Computer Studies, Arab Open University, Saudi Arabia
}

\begin{abstract}
Steganography refers to the manner in which secret data is concealed under a non-secret data. This non-secret data is known to be a digital form of media that are used as a cover. There are extensive researches regarding the images of steganographic methods. Nevertheless, the cover files did not receive enough attention. This study aims at providing a more inclusive approach for video steganography method. The difference in video steganography lies in the fact that they allow both sender and receiver to exchange hidden data through the matching between the secret text and the video frame RGB channels values presented in pixels, and manageable through different sizes of video files. The traditional manner contains the secret data under the video itself. The proposed method is designed over multithreading technique (parallelized method) for performance enhancement purposes. The results show that this developed steganographic method is more secure compared to the traditional steganographic method. In addition, results show that parallelism has impacted the proposed method positively.
\end{abstract}

Keywords - Steganography, Parallelism, Multithreading, Exact Matching Algorithm.

\section{Introduction}

High-performance computing is progressively in demand in many day-to-day applications.

DOI: $10.18421 /$ TEM92-24

https://doi.org/10.18421/TEM92-24

Corresponding author: Sally Almanasra, Arab Open University.

Email: s.almanasra@arabou.edu.sa

Received: 06 November 2019.

Revised: 27 April 2020.

Accepted: 30 April 2020.

Published: 27 May 2020.

(c) BY-NC-ND (C) 2020 Sally Almanasra; published by UIKTEN. This work is licensed under the Creative Commons Attribution-NonCommercial-NoDerivs 4.0 License.

The article is published with Open Access at www.temjournal.com
Current computing resources present a tremendous opportunity for creating higher performance models through parallelism. The main concept of parallelism relies on allowing several tasks to be accomplished simultaneously and completed in a shorter period of time. Concurrent use of multiple processing resources is able to solve complex computational problems. A given problem is broken into smaller portions and solved concurrently using multiple computing units. To obtain the best of parallelism, scientists are focusing on faster hardware devices and processing techniques [1], [2].

When exchanging information that are of a high value, there is a risk of being hacked or interrupted. The risk may be fatal in different fields as those of military and industry. Such data exchanging has to not rely on the existing traditional data transfer manners, instead, high secure methods are needed to satisfy complex security requirements. For this purpose, the science of steganography is found. Steganography is a strategy for exchanging data based on hiding the secret data under a cover from a digital media type. This digital media may be images, texts, audios or videos in which the sender and receiver agree on a stego-key to be used when extracting the data. There are many strict characteristics that have to be available in any secure steganographic method in order to be considered as secure. These characteristics include: imperceptibility/invisibility, payload/capacity, and robustness.

In this paper we propose a parallel video steganography method that does not embed the secret data in the cover file unlike the traditional steganography methods and techniques. It is due to the fact that this technique locates the exact matches between the video frame pixels itself (RGB value), and the secret text. As a part of the secret text extracting process, a stego-key is generated as a random Key-Dependant Data.

The rest of the paper is organized as follows. Section 2 provides an overview of parallelism over multi-core processors. Section 3 discusses the related works on steganography. The design and structure of the parallel steganographic method is introduced in Section 4. A number of performance tests were 
performed and results are presented in Section 5. A concluding remark is given in Section 6.

\section{Parallelism over Multi-core Processors}

Multi-core processors are widely used by individuals and enterprises. Software which is designed on a sequential base has become obstructive to performance. In order to overcome the deficiency of sequential algorithm and utilize the extra cores, new algorithms have to be designed in parallel bases. Such parallel designs effectively facilitate the utilization of multi-core processors.

Parallelism can be presented in the form of threads. This technique maps independent tasks to threads at the lowest level [3]. Multithreading techniques enhance the performance of the running processes by allowing proper distribution of tasks among the available cores in a particular computing unit [4].

Multithreading allows algorithms to execute several instructions per cycle, resulting in higher processor utilization and significant throughput speedup. Applying parallel techniques in information security has become essential for higher throughput and improved performance, especially with the current available resources. Therefore, in this study, we utilize multi-core technology with multithreading techniques to speed up the steganographic processes, in order to provide secure and better-performing security systems.

\section{Related Works}

Two methods of steganography that are relatively considered are the video steganography randomization and parallelization [5]. As for this method, it processes data before the embedding process using a frames that are randomly chosen to generate a specific key with no repeated frames to be used as the Feedback Shift Register (FSR) eliminates any repeated frames in the embedding process using a pseudo-random number generator. The data is encrypted by dividing it into equal parts, each known as $(m)$. These parts are then XOR'ed with the secret key.

In the next step, the result value is concealed through a method known as: Least Significant Bit (LSB). The result is concealed inside the frames which were selected before randomly. In the extraction process, these selected frames are identified by pseudo-random number generator and by the FSR. The results show that this method is not secure enough against security attacks due to the fact that LSB method modifies the values of the pixels.

In [6], the authors present a technique of video/image steganography. This technique encrypts the secret message before starting the embedding process. A technique known as DE-interlaces the video file and converts the images to the format of Joint Photographic Experts Group (JPEG). In this format, every image is divided into blocks of size (3 by 3) pixels. In this step, the technique scans each block to find the minimum pixel value in each block $\left(\mathrm{P}_{\min }\right)$ which enables the technique from making some modification to the block pixels' value. These modifications are made through finding the distance which is symbolized as $(\mathrm{d})$ between $\left(\mathrm{P}_{\min }\right)$, and every pixel value $(\mathrm{P})$ within the same block, by subtracting $\left(\mathrm{P}_{\text {min }}\right)$ from each pixel at the block $\left(\mathrm{d}=(\mathrm{P})-\left(\mathrm{P}_{\mathrm{min}}\right)\right)$. From another perspective, the same technique searches for the maximum value of (d) for each block $\left(\mathrm{d}_{\max }\right)$. Next, a bit by bit insertion is carried out and swaps $(\mathrm{N})$ with the rightmost bits to $(\mathrm{N})$ leftmost places. As a result, the quality of the stego-video file and the image are found to be low.

In [7], the author represents a new video steganographic method based on the non-uniform rectangular partition of the secret video in which the secret video is concealed under another video stream of nearly the same size. These obtained partitioned codes are encrypted versions of the original video frame. In the host video frames, these partitioned codes are found hidden in the least 4 significant bits without any obvious change or needed modification in the hosting or cover video as any changes in the host video after merging both the hidden, and the host video may and will affect the video quality. As a result, the invisibility and the robustness characteristics of the method will be affected.

A parallel steganographic framework is presented in [8]. The framework is designed to hide a color image inside stereo images. The underlying design is based on Discrete Cosine Transform (DCT) and Quantization Index Modulation-Dither Modulation (QIM-DM). The platform uses the Arnold's Cat Map Transform to scramble the secret color image to guarantee better security and robustness of the proposed system. The experiment results show that framework has demonstrated better performance compared to other approaches. These results emphasis that parallelism (presented in the form of multithreading) is an effective approach for increasing the processing speed.

\section{The Parallel Video Steganographic Method}

As shown in Figure 1, our proposed video steganographic method consists of two stages showing the roles of both the sender and the receiver. For the sender, the video (cover media) is selected and the frames are extracted. At the same time, the secret text which needs to be covered is specified. Then, converting the secret text into its ASCII code representation within the selected frames. 
Consequently, the method finds the exact match between the RGB values and the to-be-covered text presented as ASCII codes. This phase generates a list of the matched values of pixels RGB respectively with the location (index) of each character of the secret text. This list is necessary for formulating the stego-key as Random Key-Dependent Data (RKDD), which represents the next step. The stego-key generator generates the stego-key, and then compress it to a less size using the Arithmetic encoding technique and this key is to be shared with the receiver only. On the receiver side, the video file and the stego-key are used to find the exact marching between the RKDD stored in the decoded stego-key and the RGB values located in the extracted video frames.

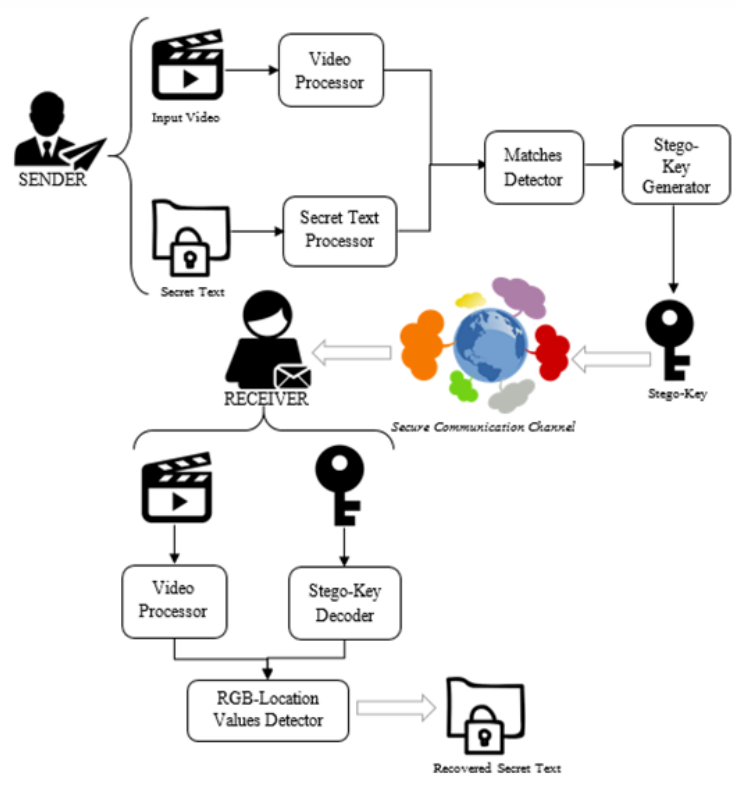

Figure 1. The Structure of the Proposed Scheme.

The Audio Video Interleaved (AVI) file is the format used in our method. It is used as an input video due to the fact that it is a popular multimedia format which is widely used. As for the frames of the video, they are extracted and saved as a portable network graphics (png) file format for its high conservation ability when compressing data. The method uses the reference frame extraction technique to extract the key frame and generate a reference frame [9], [10]. This process is preferred for its simplicity presented in only comparing the frames in the shot with the reference frame to generate the key frame as in [11], [12].

To select these frames randomly, we use a Pseudorandom Number Generator (PRNG). It generates a sequence of numbers. When the same input is given, the same output is resulted. The Lehmer PRNG is the most used technique for generating a number sequence in the current time.
The LPRNG deals with three integer parameters presented as $(r, t, n)$ and an initial seed value $\left(x_{0}\right)$ as defined in Equation (1)[13]:

$$
X_{n+1}=\left(r x_{n}+t\right) \bmod n
$$

Where, $x_{n}$ represents a particular number in the sequence, $n$ is a prime integer, and $(r, t)$ are both integers between 2 , and $n-1$. The multipliers $(r, t)$ and the prime modulus $(n)$ are selected accurately for a maximum length sequence. Upon completing the extraction process, the method starts in processing the entire number of frames as modulus $(n)$ as the length of the sequence is $(n-1)$. As a result, the entire extracted frames are to be included in selection. To avoid the repetition, the first sequence is taken by excluding the last number $(n-1)$.

In the next step, the secret data is divided into different segments in a number that matches the number of extracted frames to reduce the search time with respect to including the entire characters in the search process. In case there is a missing character in a frame, then it is in the other frame. Once the search process is complete, the entire matches are discarded of the repeated characters. One match is taken for each repeated character. Lastly, once the total length of the secret text is calculated, it is divided according to the length of characters $(c)$ over the number of the estimated segments $(s)$, as in Equation 2:

$$
s=c /(y-1), \quad \mathrm{s}(y-1)<c
$$

in which $y$ refers to the number of selected frames.

In this phase, the length of characters has to be divided exactly by the number of selected frames. Otherwise, the process will end up with extra smaller segments. Once this phase is done successfully, we assign data segment to a selected frame in order to find the matches between the secret text and the frames (RGB) values. Multithreading technique is adopted to reduce the search time and enhance the method's performance. Randomization disables any intruder from anticipating the frames pertaining to the randomization process.

After finding the exact match between the frames and the secret message the demand for reading the frames (RGB) values as a form of decimal values stored in a single dimensional matrix comes as a task. As a result, the secret text is converted to the ASCII representation, and to find the exact match, we use the brute force algorithm. In this stage, parallelism is adopted. The matching between the secret data segments and the video frames is done in parallel. Figure 2 represent the parallelized search process for the matches between the segments and the frames. 


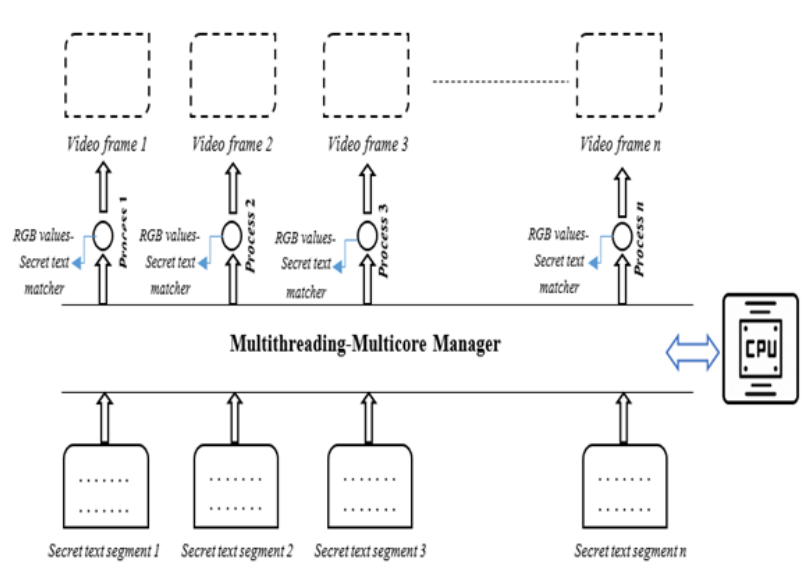

Figure 2. The search process for the matches between text segments and video frames

The method depends on a multithreadingmulticore manager as a middle layer between the segments and the frames. This manager is responsible for detecting the number of cores which are available on the users' machine, and then generate multiple threads that are associated with these cores. In the search process, the method returns the subsequent values as a list, the characters with their ASCII code representation, and its index in the secret text. If matches are not found for a particular character in the selected frames, the color channel is changed to Red and the search process start all over again.

Once a character is found to be matched, it is removed automatically from the search list. Then, we move into the next one. This method - when facing repeated character in the secret text (referred to as ( $m$ ) times) - does not save its location as the method considers it to be only one match from the frame RGB values. The locations of the entire matched locations $(x, y)$ in the frame and in the secret text are stored in an array only when the search is completed. Table 1 shows a sample search of some secret text characters "go for tests" in a video.

According to the data presented in Table 1, the length of the secret text is 12 characters. The characters (o), (space), (t) and (s) are repeated several times in the secret text. In this step, the new method selects one matching value randomly and stores it as a representative of the entire repeated characters including the key generation process which positively affect the processing time.

The most important factors in the improved method are: the key generation and the key size process as they both presented the source of many issues in the traditional method. In our improved method, we use a new approach which depends on utilizing the matching pixels in the host video frames and the secret data respectively.
Table 1. Sample search process to find matching values for secret data "go for tests"

\begin{tabular}{cccc}
\hline $\begin{array}{c}\text { Coordinates } \\
(\mathbf{x}, \mathbf{y})\end{array}$ & $\begin{array}{c}\text { Secret } \\
\text { Character }\end{array}$ & $\begin{array}{c}\text { Location of } \\
\text { Secret } \\
\text { Character }\end{array}$ & $\begin{array}{c}\text { Frame } \\
\text { Number }\end{array}$ \\
\hline$(5,90)$ & $\mathrm{g}$ & 1 & 5 \\
$(0,121)$ & $\mathrm{o}$ & 2 & 3 \\
$(3,15)$ & space & 3 & 2 \\
$(0,88)$ & $\mathrm{f}$ & 4 & 4 \\
$(0,121)$ & $\mathrm{o}$ & 5 & 6 \\
$(53,23)$ & $\mathrm{r}$ & 6 & 1 \\
$(3,15)$ & space & 7 & 1 \\
$(3,141)$ & $\mathrm{t}$ & 8 & 6 \\
$(0,23)$ & $\mathrm{e}$ & 9 & 2 \\
$(65,98)$ & $\mathrm{s}$ & 10 & 8 \\
$(3,141)$ & $\mathrm{t}$ & 11 & 1 \\
$(65,98)$ & $\mathrm{s}$ & 12 & 2 \\
\hline
\end{tabular}

In the secret text, each character has a location in the text represented in an array, and the array index shows all locations. The key holds four basic values for each character as follows: the first value represents the matching pixels locations $(x, y)$ in the cover-video frames. The second value represents the frame number for which the matching pixels belong. The third value represents the character location of the secret text. Lastly, the fourth value represents the number of digits for each element on the key. Each digit is separately represented in the key, in which the key structure is performed before the start of the compression process.

On generating the key, the improved method uses an alternative approach which represents the key values in their proper order. During the key generation process, the number of digit for all encountered value is represented. The first element of the key represents the $x$-axis value, where this value is composed of two parts: the number of digits for the $x$-axis, and the $x$-axis value itself. The digits may be one or two. The most critical values are: the character and the location of the secret text. Upon completing the character locations, there are no more character matches for the $x$-axis and the $y$-axis values. This scheme is capable of processing a huge number of repeated characters in the same frame, from one perspective. From another perspective, it is capable of representing an unlimited number of characters in a given stego-video.

Using the Arithmetic coding compression technique is the following step in this method. It is used to compress the Stego-key to reduce the large key size. In this step, the input of the compression process is the output of the search process. In Figure 3 , we present a parallelized model for the stego-key generation process. 


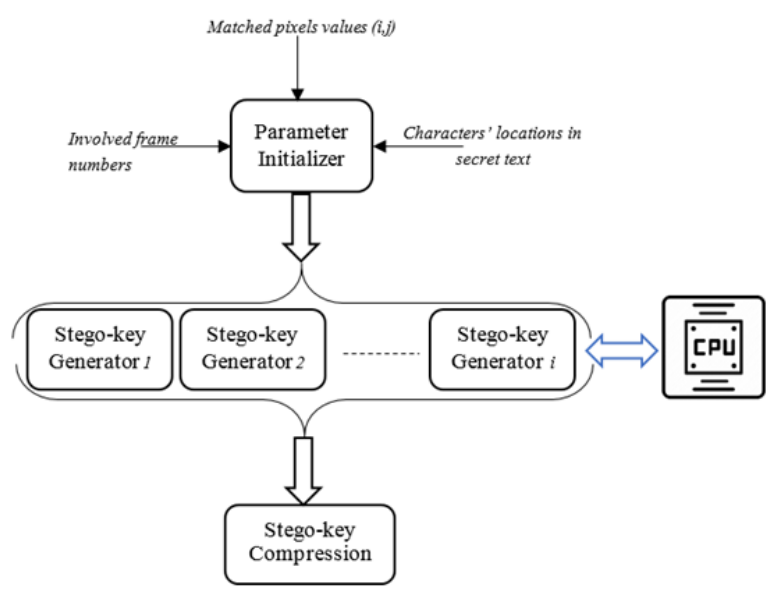

Figure 3. Parallel model for stego-key generation

The process of generating the stego-key is carried out in parallel mode. Multiple generators are created to generate the stego-key using multithreading technique. The generators work over a set of initial parameters including: the involved frame numbers, the characters' location in the secret text, and lastly the matched pixels values.

At the receiver side, the extraction process depends on decoding the stego-key. We use the cover video as input to the extraction process. Then, reading the first four bits referring to the number of the $x$-axis digits. After adding the $x$-axis digits and values accordingly, we continue to the next four bits to recover the $y$-axis data including numbers and values. For the key recovery, we add each data including frame numbers and each character. We repeat this step frame by frame. Upon competition, the method will build the secret text by converting the pixel values to the corresponding ASCII code. The process of decoding the stego-key and extracting the hidden text through is as illustrated by Figure 4 .

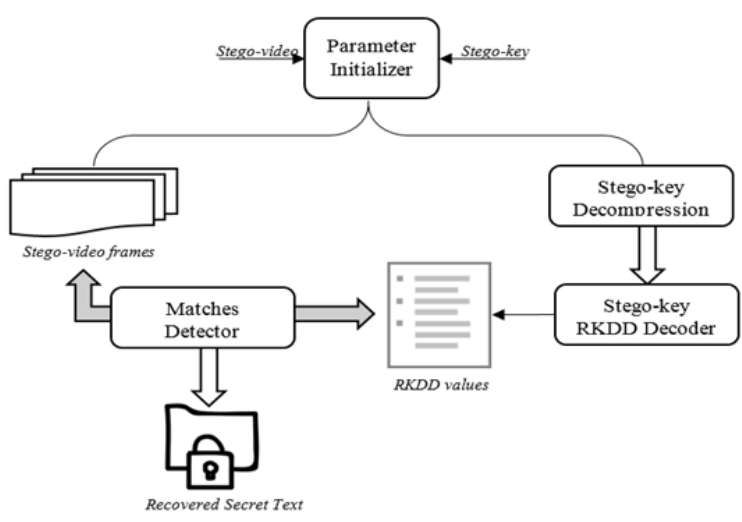

Figure 4. The process of decoding the stego-key and recovering the secret text

\section{Results and Discussions}

The tests' results show that our alternative method is reliable, secure and efficient from several aspects.
First, there are no needed modifications made to the cover video. As a result, the visibility feature is well considered in our new video steganographic method. Second, the frames in the embedding process - being selected randomly- proves that there will be always enough matches for each character but only one of them is selected according to the $(x, y)$ values. Lastly, the Stego-key is generated randomly based on the matches locations and characters in the text to be hidden which permits no possible predicting to ensure the highest possible security level.

In this paper, the proposed steganographic method is tested according to the benchmark tests. The later includes: the capacity, invisibility, robustness, and security. The method is also tested onto videos from a wide range of sizes from one perspective. From another perspective, it is tested on a wide range of secret text sizes.

For each test, only three frames are selected from the video. As for the secret message sizes, they vary between 3 and 2000 characters. In Table 2, the number of matches of the secret message is mentioned in the corresponding videos (referred to as StegoV1, StegoV2 and StegoV3).

In Table 3, a statistical analysis for the number of matches is presented. The analysis aims at finding any statistical relationship between the sizes of both: the video size and the secret texts in multiple cases. The Coefficient of Variation (CV) is proven by the tests results to be less than 1 for all the secret texts. As a result, low statistical correlations are found to enable using any size of both cover video and hidden text.

Table 2. Number of Matches of Different Secret Texts in Different Videos

\begin{tabular}{|c|c|c|c|}
\hline $\begin{array}{c}\text { Video } \\
\text { File } \\
\end{array}$ & & $\begin{array}{c}\text { No. of Secret Text } \\
\text { Characters }\end{array}$ & $\begin{array}{c}\text { No. of Matches } \\
\text { of Secret Text }\end{array}$ \\
\hline 1 & \multirow{6}{*}{ 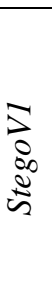 } & 3 & 15,369 \\
\hline 2 & & 10 & 41,101 \\
\hline 3 & & 100 & 299,953 \\
\hline 4 & & 500 & 51,215 \\
\hline 5 & & 1000 & 241,989 \\
\hline 6 & & 2000 & 265,111 \\
\hline 7 & \multirow{6}{*}{ 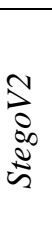 } & 3 & 32,109 \\
\hline 8 & & 10 & 21,252 \\
\hline 9 & & 100 & 151,998 \\
\hline 10 & & 500 & 61,251 \\
\hline 11 & & 1000 & 71,211 \\
\hline 12 & & 2000 & 79,742 \\
\hline 13 & \multirow{6}{*}{ 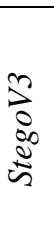 } & 3 & 24,894 \\
\hline 14 & & 10 & 50,011 \\
\hline 15 & & 100 & 233,514 \\
\hline 16 & & 500 & 58,589 \\
\hline 17 & & 1000 & 159,899 \\
\hline 18 & & 2000 & 161,021 \\
\hline
\end{tabular}


Table 3. Statistical Results on the Number of Matches of Different Secret Texts in Different Videos

\begin{tabular}{ccccc}
\hline $\begin{array}{c}\text { Secret } \\
\text { Text } \\
\text { Size }\end{array}$ & StegoV1 & StegoV2 & StegoV3 & C.V. \\
\hline $\mathbf{3}$ & 15,369 & 32,109 & 24,894 & 0.28419 \\
$\mathbf{1 0}$ & 41,101 & 21,252 & 50,011 & 0.66627 \\
$\mathbf{1 0 0}$ & 299,953 & 151,998 & 233,514 & 0.26481 \\
$\mathbf{5 0 0}$ & 51,215 & 61,251 & 58,589 & 0.07445 \\
$\mathbf{1 0 0 0}$ & 241,989 & 71,211 & 159,899 & 0.44222 \\
$\mathbf{2 0 0 0}$ & 265,111 & 79,742 & 161,021 & 0.44991
\end{tabular}

The results of searching for matches are determined by the number of characters as this number is used in the corresponding hidden text. Our method works on skipping any repetition of characters within the search process. Regardless the number of repetition, we use only one match per character. On a larger scope, the secret text is divided into segments, and we use one segment per frame.

In term of performance, we measure the speed of our method in finding the matches of the hidden secret with regard to the diversity of size. Our experiment is carried out over a personal computer of Core i7 CPU $(2.4 \mathrm{GHz})$ with 6GB RAM. As for the cover videos, the same three stego videos (StegoV1, StegoV2 and StegoV3) are considered.

In this experiment we implement our method in both sequential and parallel modes. The purpose of such implementation is to measure the efficiency of utilizing the processing power available on computers nowadays. The results are shown in Figures 5-7 displays that our parallelized method enhances the performance of the sequential method of about $45 \%$. The results are applied over a secret text of sizes range from 10 to 2000 characters. The same secret text is considered with three different stego-videos. As a result, we found that the differences between the used stego-video have not affected the performance of the methods significantly.

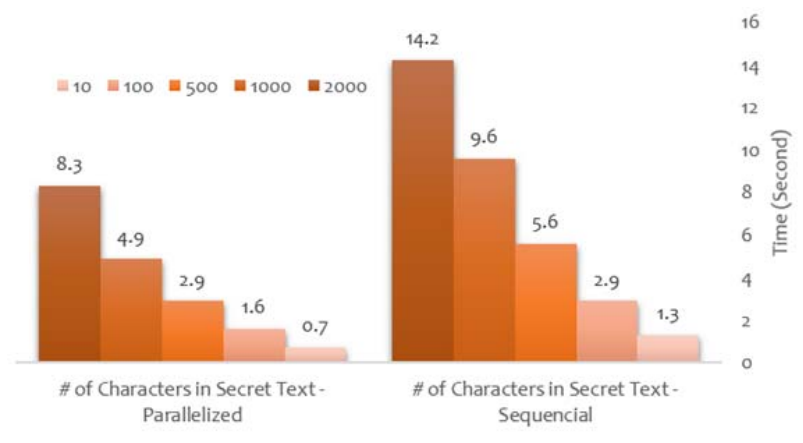

Figure 5. Performance evaluation for finding matches using secret texts of different sizes over StegoV1

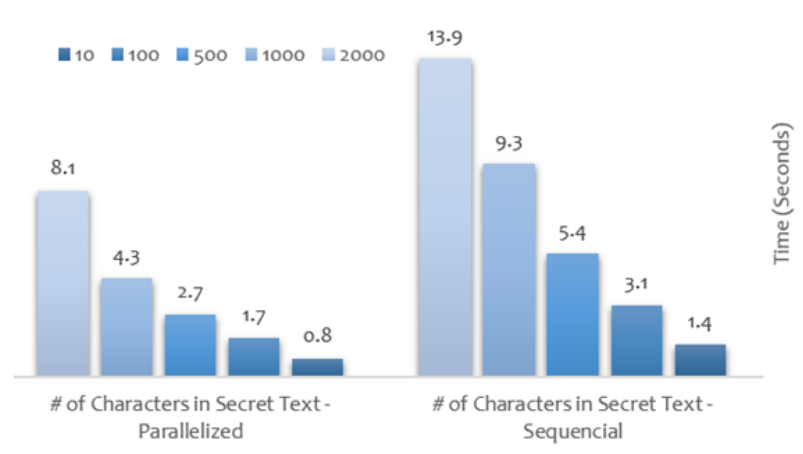

Figure 6. Performance evaluation for finding matches using secret texts of different sizes over StegoV2

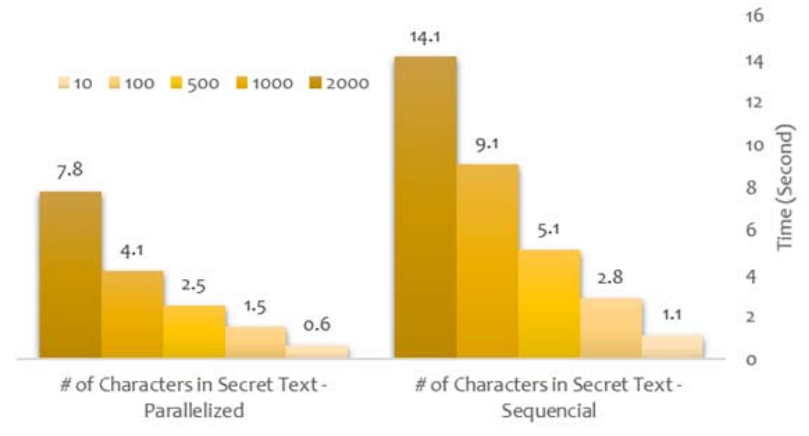

Figure 7. Performance evaluation for finding matches using secret texts of different sizes over StegoV3

To evaluate steganographic primitives, we use two metrics values presented in fidelity and quality [14], [15]. Fidelity determines the similarity between the host video and the stego-video. To find the key, in our improved method, we find matches through inputting the host video. Unlike the traditional method, the receiver gets the unmodified original video. As for quality, it is the factor that determines integrity for the video as it can neither be modified nor changed.

Fidelity metrics represent; the Peak Signal to Noise Ratio (PSNR) and the Mean Error Square (MES). The PSNR measures the similarity between the cover and the stego videos. If the PSNR value is high, then both videos are $100 \%$ similar, and vise-versa as the original video represents the stego-video. Our scheme makes no modifications to any values of any pixels at all. The tests' results show that the MSE condition is achieved.

Another important characteristic for the steganographic primitives is Robustness. It measures the defence ability against hacking attacks whether they are visual or statistical. Visual attacks target any modifications in the video before embedding and after, and since no modifications are made, they cannot be detected. The same can be said regarding the statistical attacks as there is no modification made at all. In brief, there are no possible attacks against our improved steganographic video method.

The accuracy tests show that our method decodes the secret text with $0 \%$ error throughout all video and 
text sizes. Figure 8 shows the time taken to recover different secret texts using the three previously mentioned stego-videos.

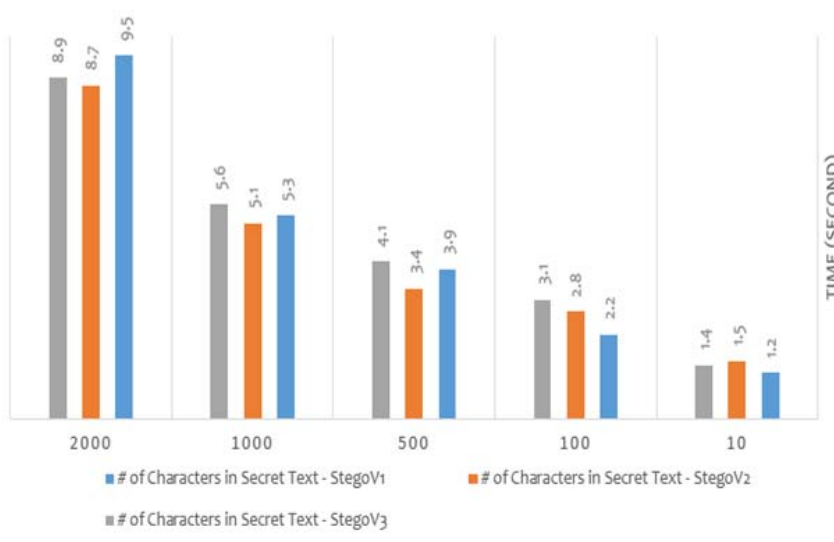

Figure 8. Recovery time of the secret text using three different stego-videos

\section{Conclusion}

This paper has presented an improved parallel steganography video method. The method extracted the data through the match between the secret texts in the form of ASCII codes from one perspective, and between the cover video frame itself in the form of RGB values from another perspective as well. Parallelism is utilized to enhance the performance of the matching process. Multiple threads are generated according to the available cores in the sender's/receiver's machine.

As for the capacity, the results have shown that our developed method is capable to find the matches of any hidden message regardless its size. The experimental tests prove that our improved steganographic method has managed to deal with a text whose size is estimated as $(700,000)$ characters. Our method is also untracked since cover video is unmodified allowing no hackers to track any modifications.

In term of visibility, our method managed to achieve high invisibility. The stego-key is considered between the sender and receiver to add an extra level of security through. As for the processing time, it takes around 3.6 milliseconds to uncover one character of the hidden text.

\section{References}

[1] Nutaro, J., \& Zeigler, B. (2017). How to apply Amdahl's law to multithreaded multicore processors. Journal of Parallel and Distributed Computing, 107, 1-2.

[2] Dang, H. V., Snir, M., \& Gropp, W. (2017). Eliminating contention bottlenecks in multithreaded MPI. Parallel Computing, 69, 1-23.

[3] Hiscock, T., Savry, O., \& Goubin, L. (2019). Lightweight instruction-level encryption for embedded processors using stream ciphers. Microprocessors and Microsystems, 64, 43-52.

[4] Lai, B. C. C., Li, K. C., Li, G. R., \& Chiang, C. H. (2015). Self adaptable multithreaded object detection on embedded multicore systems. Journal of Parallel and Distributed Computing, 78, 25-38.

[5] Sudeepa, K. B., Raju, K., Ranjan, K., \& Ghanesh, A. (2016). A new approach for video steganography based on randomization and parallelization. Procedia Computer Science, 78, 483-490.

[6] Elbayoumy, M., Elmogy, M., Abouelfetouh, A., \& Elhadary, R. (2014). A Proposed Technique For Hiding Data Into Video Files. International Journal of Computer Science Issues (IJCSI), 11(2), 67-78.

[7] Hu, S. D. (2011, August). A novel video steganography based on non-uniform rectangular partition. In 2011 14th IEEE International Conference on Computational Science and Engineering (pp. 5761). IEEE.

[8] Munoz-Ramirez, D. O., Ponomaryov, V. I., ReyesReyes, R., \& Cruz-Ramos, C. (2017, May). Parallel steganography framework for hiding a color image inside stereo images. In Real-Time Image and Video Processing 2017 (Vol. 10223, p. 102230L). International Society for Optics and Photonics. https://doi.org/10.1117/12.2262163.

[9] Nasreen, A., \& Shobha, G. (2013). Key frame extraction from videos-A survey. International Journal of Computer Science \& Communication Networks, 3(3), 194-198.

[10] Rosaline, S. I., \& Raj, M. A. (2013, January). Adaptive pixel pair matching based steganography for audio files. In 2013 International Conference on Emerging Trends in Vlsi, Embedded System, Nano Electronics and Telecommunication System (Icevent) (pp. 1-5). IEEE.

[11] Manjula, R. and Danti, A., (2015). A Novel Hash Based Least Significant Bit (2-3-3) Image Steganography in Spatial Domain, International Journal of Security, Privacy and Trust Management (IJSPTM), 4(1), 11-20.

[12] Gupta, R., Gupta, S., and Singhal, A., (2014). Importance and Techniques of Information Hiding: A Review, International Journal of Computer Trends and Technology, 9(5), 260-265.

[13] Oduwole, K., Shehu, L., Adegoke, K., and Onubogu, L. (2013). Fibonacci Random Number Generator using Lehmer's Algorithm. Mathematical Theory and Modeling, 13(14), 56-61.

[14] Apau, R. (2017). Enhancing Data Security using Video Steganography, RSA and Huffman Code Algorithms with LSB Insertion (Doctoral dissertation).

[15] Sloan, T., \& Hernandez-Castro, J. (2015). Forensic analysis of video steganography tools. PeerJ Computer Science, 1, e7. 